\title{
Investigation of the shear-mechanical and dielectric relaxation processes in two monoalcohols close to the glass transition
}

\author{
Bo Jakobsen, ${ }^{\text {a) }}$ Claudio Maggi, Tage Christensen, and Jeppe C. Dyre \\ DNRF Centre "Glass and Time," IMFUFA, Department of Sciences, Roskilde University, Postbox 260, \\ DK-4000 Roskilde, Denmark
}

(Received 29 May 2008; accepted 3 October 2008; published online 10 November 2008)

\begin{abstract}
Shear-mechanical and dielectric measurements on the two monohydroxy (monoalcohol) molecular glass formers 2-ethyl-1-hexanol and 2-butanol close to the glass-transition temperature are presented. The shear-mechanical data are obtained using the piezoelectric shear-modulus gauge method covering frequencies from $1 \mathrm{mHz}$ to $10 \mathrm{kHz}$. The shear-mechanical relaxation spectra show two processes, which follow the typical scenario of a structural (alpha) relaxation and an additional (Johari-Goldstein) beta relaxation. The dielectric relaxation spectra are dominated by a Debye-type peak with an additional non-Debye peak visible. This Debye-type relaxation is a common feature peculiar to monoalcohols. The time scale of the non-Debye dielectric relaxation process is shown to correspond to the mechanical structural (alpha) relaxation. Glass-transition temperatures and fragilities are reported based on the mechanical alpha relaxation and the dielectric Debye-type process, showing that the two glass-transition temperatures differ by approximately $10 \mathrm{~K}$ and that the fragility based on the Debye-type process is a factor of 2 smaller than the structural fragility. If a mechanical signature of the Debye-type relaxation exists in these liquids, its relaxation strength is at most $1 \%$ and $3 \%$ of the full relaxation strength of 2-butanol and 2-ethyl-1-hexanol, respectively. These findings support the notion that it is the non-Debye dielectric relaxation process that corresponds to the structural alpha relaxation in the liquid. (C) 2008 American Institute of Physics.
\end{abstract} [DOI: $10.1063 / 1.3007988$ ]

\section{INTRODUCTION}

A class of often investigated glass-forming liquids is the hydrogen-bonding liquids, among which the alcohols are a much studied subclass (for a compilation of references to classical results prior to 1980 see Ref. 1, Sec. IX-c.1). Alcohols are normally classified into those containing one hydroxyl group (the monoalcohols) and those with two or more hydroxyl groups.

During the 1950s it was observed that the main relaxation in most monoalcohols, contrary to the main relaxation in other liquids, can be represented by a single relaxation time-they follow the Debye prediction. ${ }^{2}$ It was further realized that additional relaxation processes exist at frequencies above the main Debye-type relaxation. One additional process is normally observed, but in some cases two processes can be resolved (see, e.g., Ref. 3). Comparisons between mechanical and dielectric measurements ${ }^{4,5}$ further showed that when the main dielectric relaxation is of Debyetype its time scale is separated from the mechanical time scale, but no explanation was given for this. It was further discussed to what extent the Debye-type process corresponds to the mechanical relaxation, as, e.g., stated by Johari and Goldstein ${ }^{6}$ discussing the importance of mechanical measurements near the glass-transition temperature; "such a study can answer an important question: whether or not the same molecular motions are involved in the volume, shear, and dielectric relaxation of $\mathrm{H}$-bonded liquids."

${ }^{a)}$ Electronic mail: boj@ruc.dk.
During the past decade a number of studies ${ }^{7-19}$ (see below for details) have indicated that the low-frequency Debye-type peak is decoupled from the mechanical relaxation and that the non-Debye dielectric peak at higher frequencies reflects the structural alpha relaxation. In this paper we shall term the two lowest frequency dielectric relaxations the Debye-type relaxation and the alpha relaxation, respectively. This scenario offers an explanation for the earlier observations and it gives the possibility that the behavior of monoalcohols follows that of other glass formers, except for the existence of the Debye-type dielectric peak.

Two classes of arguments are generally given for this idea: comparisons of time scales/glass-transition temperatures and the lack of a Debye-type peak in other measurement types. A large number of experiments and comparisons exists including the following: comparison with calorimetric measurements, ${ }^{7,8}$ comparison with photon correlation spectroscopy probing the density-density correlations, ${ }^{9}$ comparison with the time scale found from viscosity data, ${ }^{9,13}$ analysis of the alpha-beta relaxation, ${ }^{10}$ solvation dynamics probing mechanical relaxation of the liquid, ${ }^{11}$ dielectric and calorimetric investigation of mixtures of monoalcohols with other substances, ${ }^{12,14-16}$ frequency-dependent specific heat measurements, ${ }^{17}$ systematic comparison to differential scanning calorimetry measurements ${ }^{18}$ and dielectric studies of mixtures. ${ }^{19}$ Except for the early ultrasonic-based measurements $^{4,20}$ no direct comparison, to the best of our knowledge, of the macroscopic mechanical relaxation spectra and dielectric relaxation spectra of monoalcohols exists. 
Such measurements directly reveal if the non-Debye relaxation mode seen in dielectrics is in accordance with the shear-mechanical structural alpha relaxation. Shearmechanical relaxation data are, furthermore, generally a good complement to dielectric data, ${ }^{21}$ and such investigations can also explore to what extent a shear-mechanical Debye-type relaxation exists.

In this study we present shear-mechanical investigations in the temperature range down to the glass-transition temperature together with complementary dielectric spectroscopy investigation to allow for direct comparison.

The two liquids studied are 2-butanol and 2-ethyl-1hexanol. The reason for not studying simple normal alcohols like ethanol is that such systems easily crystallize. The chosen systems represent two ways of introducing steric hindrances in the system, hence improving the glass-forming ability. Both liquids have been investigated earlier. For early results on 2-butanol see, e.g., Refs. 22 and 23 and for 2-ethyl-1-hexanol see, e.g., Refs. 8, 12, 17, 18, 24, and 25.

\section{EXPERIMENTAL}

The measurements were performed using a custom-built setup. $^{26-28}$ The temperature is controlled by a custom-built cryostat with temperature fluctuations smaller than $2 \mathrm{mK}$ (see Ref. 27 for details on the cryostat). The same cryostat was used for all measurements, thus ensuring equal temperatures and directly comparable results. The electrical signals were measured using a HP 3458A multimeter in connection with a custom-built frequency generator in the frequency range of $10^{-3}-10^{2} \mathrm{~Hz}$ and an Agilent 4284A LCR meter in the frequency range of $10^{2}-10^{6} \mathrm{~Hz}$ (see Ref. 28 for details on the electrical setup).

The shear-mechanical relaxation data were obtained using the piezoelectric shear modulus gauge method. ${ }^{26}$ This method has a wide frequency range (up to $10^{-3}-10^{4} \mathrm{~Hz}$ ) and is optimized for measuring moduli in the range of $\mathrm{MPa}-\mathrm{GPa}$, corresponding to typical moduli of liquids close to the glasstransition temperature. The dielectric data were obtained using a multilayered gold-plated capacitor with a empty capacitance of $95 \mathrm{pF}$.

2-ethyl-1-hexanol ( $\geq 99.6 \%$, CAS number 104-76-7) and 2-butanol (99.5\%, CAS number 78-92-2, racemic mixture) were acquired from Aldrich and used as received. To ensure that the samples did not change characteristics (e.g., due to absorption of water), dielectric measurements were performed on the newly opened bottles and repeated at the end of the studies. For both liquids the only observable changes were in the unimportant low-frequency contributions from conduction.

The raw data ${ }^{29}$ obtained consist of frequency $\nu$, scans of the complex dielectric constant $\epsilon(\nu)$, and the complex shear modulus $G(\nu)$. Each scan was taken at constant temperature in thermal equilibrium, stepping down in temperature.

Equilibrium was ensured by repeating some of the frequency scans on reheating the sample from the lowest temperature. Repetition of parts of the shear-mechanical measurements showed that the uncertainty on the overall absolute level of the shear modulus is rather large in the case of 2-ethyl-1-hexanol $(\approx 20 \%)$; it is much better for 2-butanol. The influence of this experimental uncertainty on the position of the loss peaks is, however, minor (at most \pm 0.1 decade).

\section{RESULTS AND DISCUSSIONS}

A selection of the obtained dielectric spectra is shown in Fig. 1, represented as the dielectric loss as a function of frequency [minus the imaginary part of the complex dielectric constant $\left.-\epsilon(\nu)^{\prime \prime}\right]$. The dielectric data are further illustrated in Fig. 2 as a Nyquist plot at a representative temperature. The dielectric spectrum follows the general pattern for monoalcohols with a dominant Debye-type relaxation and a minor second relaxation - the alpha relaxation.

A common way (e.g., Refs. 3, 9, 13, 19, 22, and 23) to separate the minor alpha process and possible JohariGoldstein beta processes from the Debye-type relaxation process is to assume additivity of the processes in the dielectric susceptibility (corresponding to statistical independent dipole-moment fluctuations of the two processes). This is done either by fitting a sum of a Debye function and a Havriliak-Negami function (and possibly a Cole-Cole function for the beta process) or by subtracting the fit of a Debye function from the raw data (most common in elder studies, e.g., Ref. 3).

In this paper we assume additivity of the processes ${ }^{30}$ and subtract the Debye function in order to analyze the residual, this procedure is illustrated in Fig. 3. The fit to the Debye function is also shown in the Nyquist plot in Fig. 2, illustrating the quality of the fit with respect to both real and imaginary parts of the dominant dielectric relaxation process.

A selection of the shear-mechanical data is shown in Fig. 1 as mechanical loss $G^{\prime \prime}(\nu)$, as function of frequency. Figure 2 , furthermore, shows the shear-mechanical relaxation spectra illustrated as a Nyquist plot at a typical temperature. The general pattern for liquids close to the glass-transition temperature is observed, with a pronounced non-Debye alpha relaxation and a smaller Johari-Goldstein beta relaxation. The beta relaxation is much stronger in the shear-mechanical relaxation spectrum than in the dielectric spectrum for these liquids (the existence of a dielectric beta relaxation for these systems has been reported in the literature ${ }^{8,23}$ ), consistent with previous observation on molecular liquids ${ }^{21}$ and the Gemant-DiMarzio-Bishop model. ${ }^{31}$

\section{A. Temperature dependence of the dynamics}

To analyze the time scales associated with the observed processes and their temperature dependences, the loss-peak frequencies $\left(\nu_{\mathrm{lp}}\right)$ were determined. These are shown in Fig. 4. For the alpha process in the shear-mechanical data and the Debye-type process in the dielectric data, they were determined directly from the raw data. For the alpha relaxation in the dielectric data the loss peak was found after subtracting the Debye function. To ensure consistency in the analysis, the dielectric alpha loss peak was only calculated at temperatures where the loss peak of the Debye-type relaxation was observed. 


\section{2-butanol}
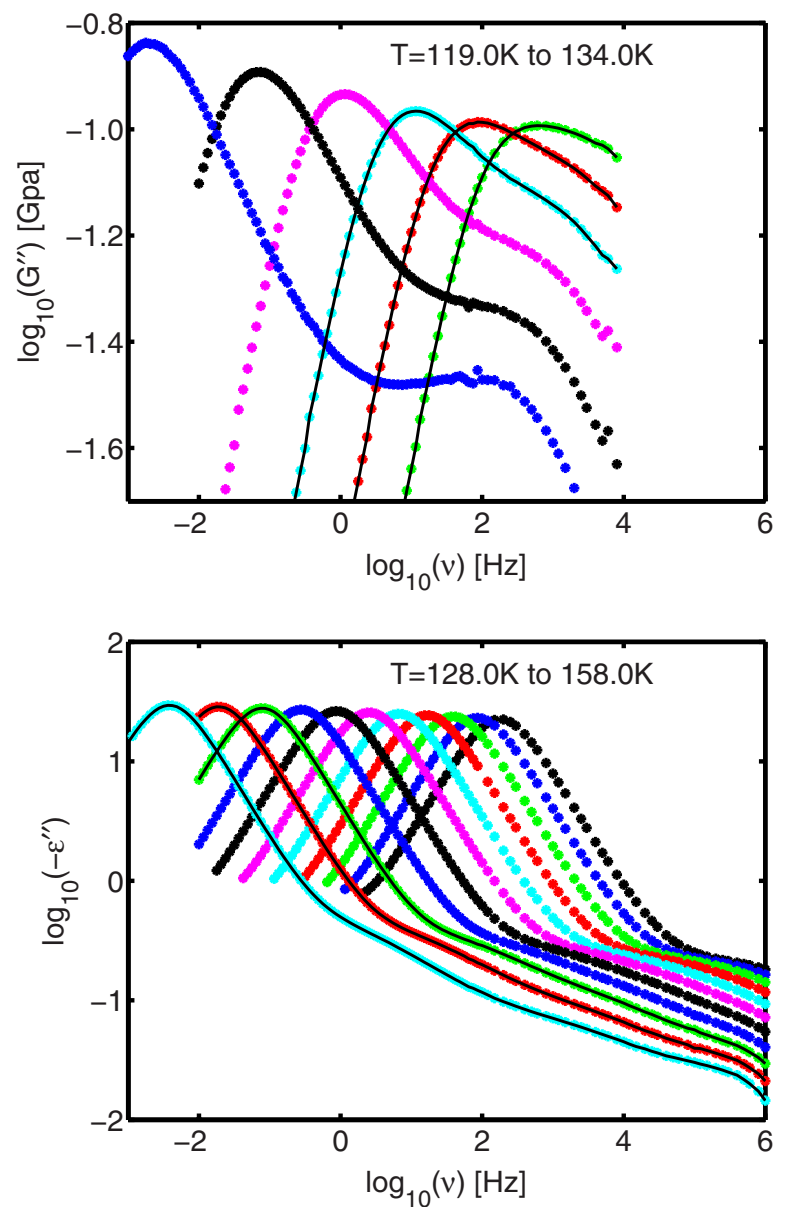

2-ethyl-1-hexanol
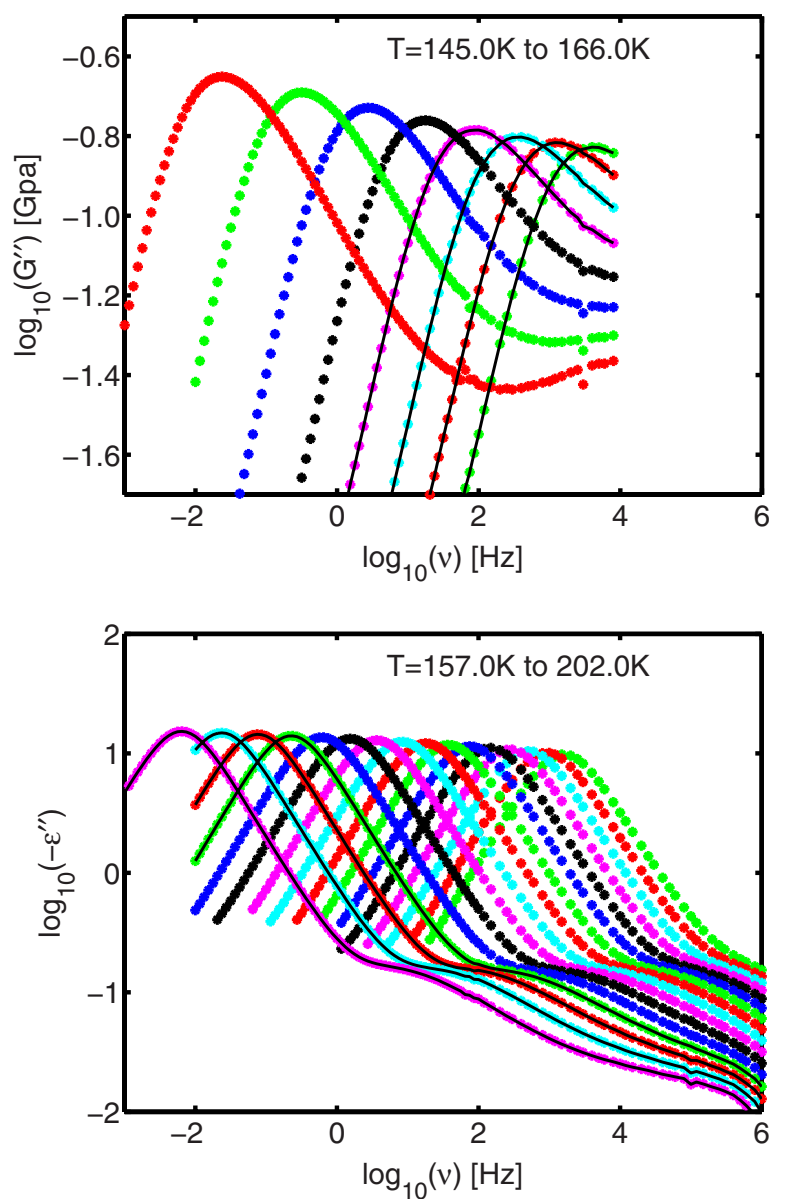

FIG. 1. (Color online) Selected dielectric and shear-mechanical spectra for the two liquids (the full data set is available online, see Ref. 29). Temperature intervals are indicated and the step size is $3 \mathrm{~K}$. Temperatures that are highlighted by a full line through the points exist for both dielectric and shear-mechanical measurements, this is only the case in a limited temperature interval, where an overlap exists between the high temperature shear data and low temperature dielectric data. Top: shear-mechanical loss as function of frequency. Bottom: dielectric loss as function of frequency. To enhance readability of the figure, the dielectric spectra have been truncated at low frequencies, at around the onset of the contribution from conduction.

The dielectric alpha-peak frequency closely follows the peak frequency of the shear-mechanical alpha process. This is further illustrated in the lower part of Fig. 4, where the "decoupling" index [defined as $\left.\log _{10}\left(\nu_{\mathrm{lp}, G} / \nu_{\mathrm{lp}, \epsilon}\right)\right]$ is shown for the dielectric processes (Debye-type and alpha process) relative to the mechanical alpha process. The mechanical and dielectric alpha-time scales are separated by approximately 1 decade in frequency, whereas the mechanical alpha and the dielectric Debye-type processes are separated by four orders of magnitude in frequency. The separation between the losspeak positions can also directly be seen in Fig. 1 for the temperatures where both shear-mechanical and dielectric data exist (indicated by full lines). The separation between the shear-mechanical and dielectric alpha processes is in agreement with previous comparisons of the shearmechanical and dielectric alpha time scale. ${ }^{4,5,21,32-36}$ From Fig. 1 it can be seen that the mechanical beta relaxation may influence the loss-peak position of the shear-mechanical alpha relaxation at high temperatures. From, e.g., Ref. 21 we know that such influences only change the decoupling index between the shear mechanic and dielectric alpha relaxations slightly; such an effect can, therefore, not disturb the general observations. It is further noticeable that no changes can be observed in the temperature dependence of the shearmechanical relaxation time around the temperature where the Debye-type process falls out of equilibrium on the time scale of the experiment.

The glass-transition temperature(s) was determined from the loss-peak frequencies ${ }^{37}$ as the temperature where $\nu_{\mathrm{lp}}$ $=10^{-2} \mathrm{~Hz}$. The numbers for the dielectric Debye-type relaxation and the shear-mechanical alpha relaxation are given in Table I. The huge separation in time scale between the two processes results in a separation of $T_{g}$ of $10 \mathrm{~K}$ for 2-butanol and $14 \mathrm{~K}$ for 2-ethyl-1-hexanol.

Based on the loss-peak frequencies an Angell plot was constructed, as shown in Fig. 5. The two substances show a remarkable similarity in the temperature dependence of the characteristic time when plotted this way. The dielectric alpha process, furthermore, closely follows the tendencies of the mechanical alpha process.

The fragility index $m=\left.\left[\left(d \log _{10} 1 / \nu_{\mathrm{lp}}\right) /\left(d T_{g} / T\right)\right]\right|_{T=T_{g}}$ (Refs. 38-40) is reported in Table I. A clear difference is seen between the fragility index if defined from the dielectric Debye-type process or from the mechanical alpha process. 


\section{2-butanol}
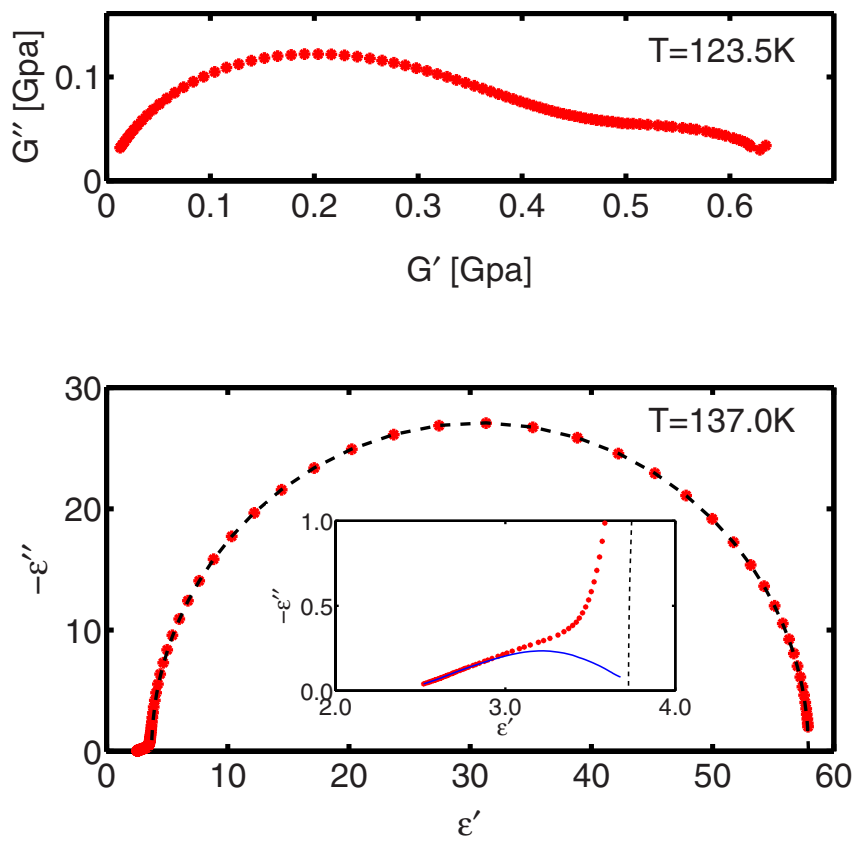

2-ethyl-1-hexanol
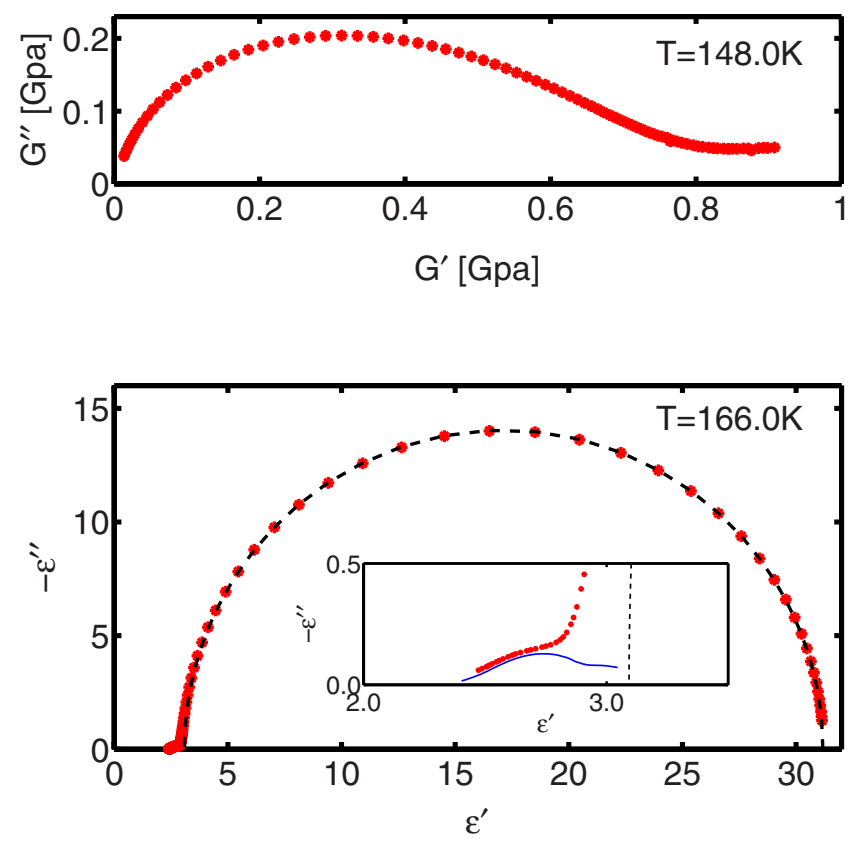

FIG. 2. (Color online) Typical dielectric and shear-mechanical spectra for the two liquids, represented as Nyquist plots. Top: shear-mechanical spectra. Bottom: dielectric spectra (the inset shows a zoom on the high frequency foot point). Lines indicate the separation of the dielectric data into a Debye-like process (dashed lines) and an alpha-relaxation process (full lines), see Fig. 3 for full explanation.

The Debye-type process leads to a classification of the liquid as much stronger than the mechanical alpha relaxation.

The difference in temperature dependence of the Debyetype process and the structural relaxation will lead to a merging of the two processes at low temperatures, if the trend continue. This is of course close to impossible to test experimentally as the relaxation times at such low temperatures become very long. The possibility of a low temperature merge of the Debye-type process and the structural alpha

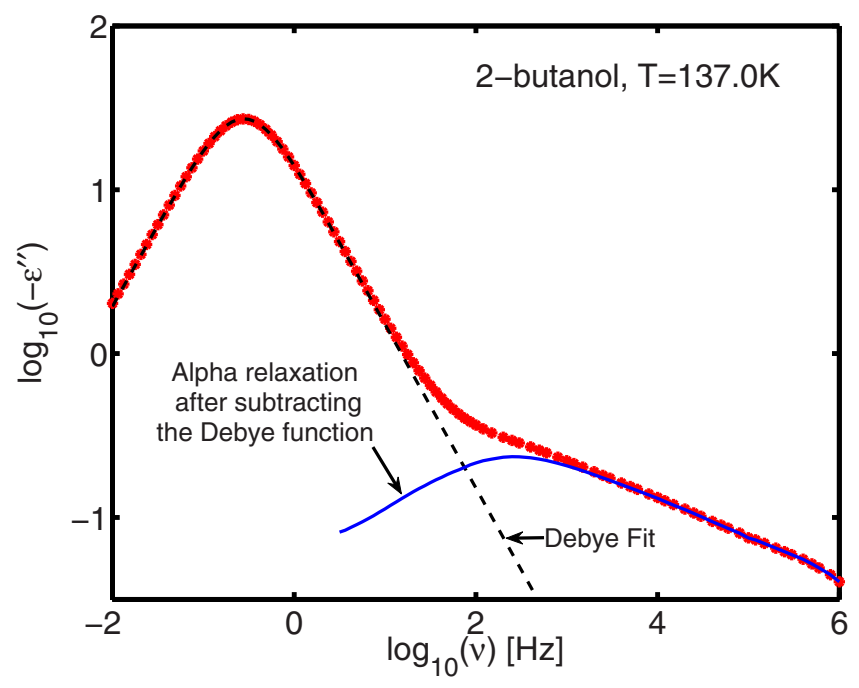

FIG. 3. (Color online) Illustration of the procedure used for separating the alpha relaxation from the Debye-type relaxation. The data shown are the dielectric loss spectrum for 2-butanol at $T=137 \mathrm{~K}$. Points are measured data, dashed line is a fit of the main relaxation to a Debye function $\epsilon=\left[\Delta \epsilon_{D} /(1+i \omega \tau)\right]+\epsilon_{D, \infty}$, and the full line the residual after subtracting the Debye function from the measured data (representing the alpha-relaxation process). process has been discussed earlier in Refs. 19 and 41. The idea is further supported by the compilation of data presented in Ref. 13; the data generally show a decrease in the difference between the loss-peak frequency of the dielectric Debye-type relaxation and dielectric alpha relaxation with decreasing temperature.

\section{B. Spectral shape}

The spectral shape of the shear-mechanical alpha peak was characterized by calculation of the minimum slope in a log-log plot, as shown on Fig. 6. Similar data have been reported for shear-mechanical relaxation studies on other systems by our group ${ }^{21,42}$ based on the ideas presented in Ref. 43. In comparing to these previous results, it is observed that the two liquids follow the general trend of liquids with a mechanical beta relaxation. The minimum slope is in the range from -0.3 to -0.4 close to $T_{g}$, still decreasing upon cooling (most prominent for 2-butanol), possibly toward -0.5 as conjectured in Refs. 44-46. This shows that the mechanical alpha-relaxation spectra, hence the mechanical relaxation processes, are similar to what is generally observed for glass-forming liquids.

\section{Limits on a mechanical Debye-type process}

A small low-frequency peak was observed in the raw data obtained by the shear-mechanical transducer. Closer investigations, however, showed that this was not a mechanical signal of the Debye-type process, but a "spillover effect" of the large dielectric signal. This effect is caused by wetting of the edges of the piezoceramic disks in the transducer and the 

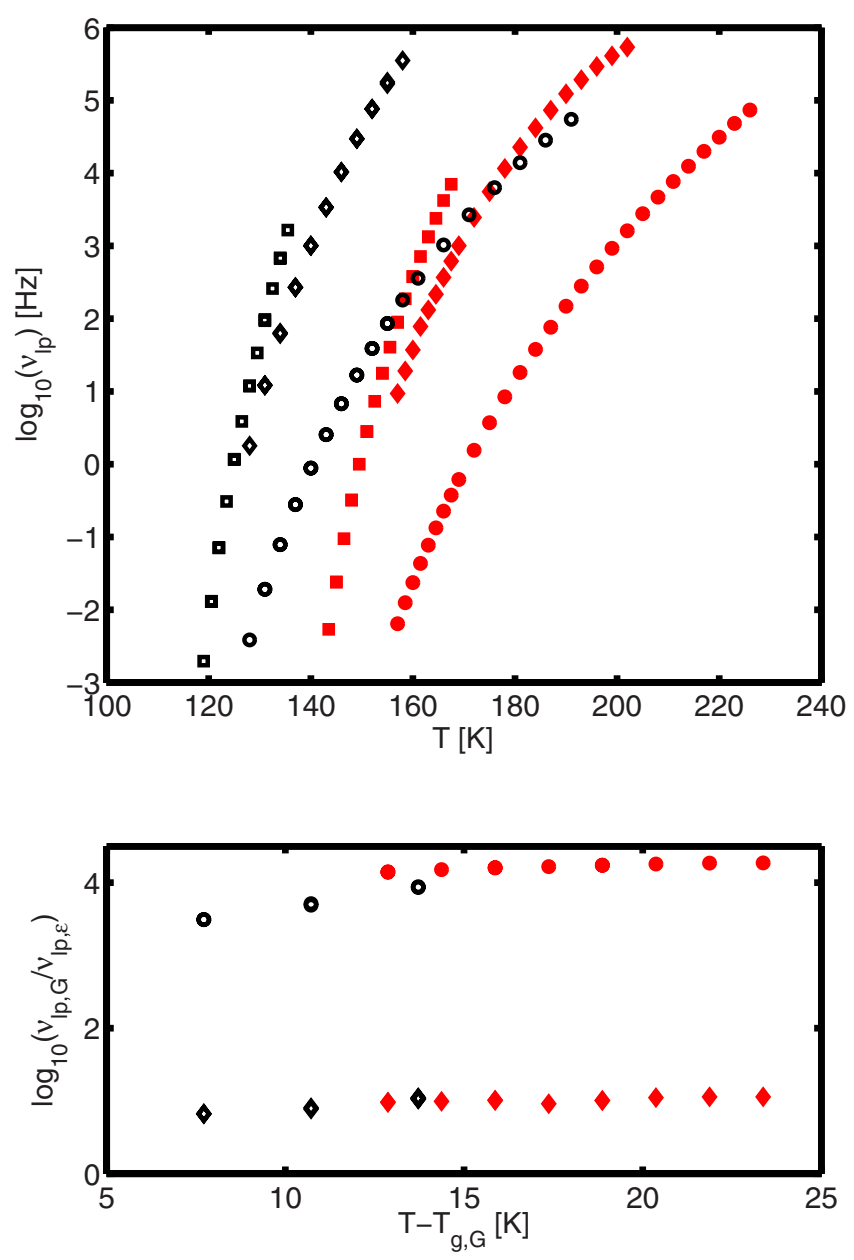

FIG. 4. (Color online) Top: loss-peak positions for the different processes evaluated for the two investigated systems (repeated measurements exist at some temperatures). Bottom: the decoupling index $\left[\log _{10}\left(\nu_{\mathrm{lp}, G} / \nu_{\mathrm{lp}, \epsilon}\right)\right]$ for the dielectric alpha and Debye-type process relative to the mechanical alpha process. ( $\square$ ) Shear-mechanical alpha relaxation. (○) Dielectric Debye-type relaxation. $(\diamond)$ Dielectric alpha relaxation. Open symbols: 2-butanol. Closed symbols: 2-ethyl-1-hexanol.

large dielectric strength of the investigated systems; it is equivalent to sometimes observed conduction contributions in the raw data.

We can, of course, not entirely exclude that a mechanical

TABLE I. Glass-transition temperature $\left(T_{g}\right)$ and fragility $(m)$ for the dielectric Debye-type process $\left(\epsilon_{\text {Debye-type }}\right)$ and mechanical alpha process $\left(G_{\text {alpha }}\right)$. The glass-transition temperature is defined from the loss-peak frequencies as $\nu_{\mathrm{lp}}\left(T_{g}\right)=10^{-2} \mathrm{~Hz}$.

\begin{tabular}{lccccc}
\hline \hline & \multicolumn{2}{c}{$T_{g}$} & & \multicolumn{2}{c}{$m$} \\
\cline { 2 - 3 } \cline { 5 - 6 } & $G_{\text {alpha }}$ & $\epsilon_{\text {Debye-type }}$ & & $G_{\text {alpha }}$ & $\epsilon_{\text {Debye-type }}$ \\
\hline 2-butanol & $120 \mathrm{~K}^{\mathrm{a}}$ & $130 \mathrm{~K}$ & & 63 & 29 \\
2-ethyl-1-hexanol & $144 \mathrm{~K}^{\mathrm{b}}$ & $158 \mathrm{~K}^{\mathrm{c}}$ & & $60^{\mathrm{d}}$ & $30^{\mathrm{e}}$ \\
\hline \hline
\end{tabular}

${ }^{\mathrm{a}}$ In accordance with calorimetric $T_{g}$ of $120.3 \mathrm{~K}$ from Ref. 23 .

${ }^{\mathrm{b}}$ In accordance with calorimetric $T_{g}$ of $145.9 \mathrm{~K}$ from Ref. 18 and of $148.9 \mathrm{~K}$ from Ref. 8, and dielectric alpha-relaxation $T_{g}$ of $144.0 \mathrm{~K}$ from Ref. 18.

${ }^{c}$ In accordance with the value of $155.0 \mathrm{~K}$ reported in Ref. 18, and $154.0 \mathrm{~K}$ reported in Ref. 8 [using $\nu_{\mathrm{lp}}\left(T_{g}\right)=10^{-3} \mathrm{~Hz}$ as definition of $T_{g}$ ].

${ }^{\mathrm{d}}$ Comparable to the value of 70 reported in Ref. 18 on the basis of dielectric data.

${ }^{\mathrm{e}}$ In accordance with the value of 27.0 reported in Ref. 8 .

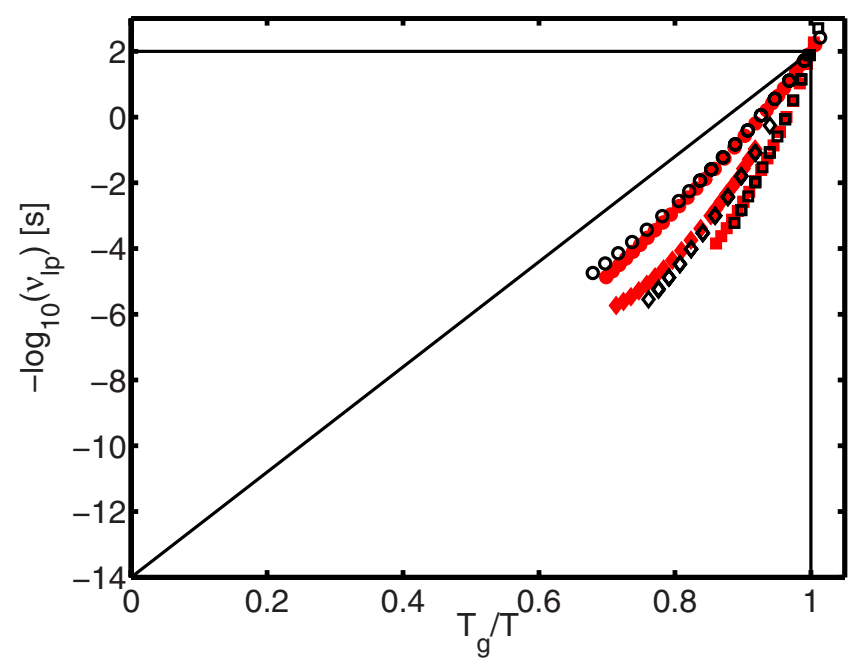

FIG. 5. (Color online) Angell fragility plot based on loss-peak positions. (Symbols as in Fig. 4.) The vertical and horizontal lines define the glass transition, the diagonal line corresponds to Arrhenius behavior. $T_{g}$ for the mechanical alpha relaxation and dielectric Debye-type relaxation is as given in Table I. For the dielectric alpha relaxation, $T_{g}$ from the mechanical alpha relaxation was used. The reason for this is that the data do not allow for direct determination of the $T_{g}$ for the dielectric alpha relaxation without extensive extrapolation, and that the two temperatures normally are not too different due to the small decoupling between the processes.

signal is hiding below this dielectric spillover signal, but we are able to put limits on the maximal relaxation strength. In the case of 2-butanol the signal was partly eliminated by a correction procedure using data from a mechanically empty but still wetted transducer. From the resulting shearmechanical spectra one can conclude that a shear-mechanical relaxation process corresponding to the Debye-type process in the dielectrics must have a relaxation strength below 5 $\mathrm{MPa}$ (corresponding to at most $1 \%$ of the full relaxation strength) if it exists. In the case of 2-ethyl-1-hexanol the raw data show that a mechanical Debye-type relaxation process must have a strength below $30 \mathrm{MPa}$ (corresponding to at most $3 \%$ of the full relaxation strength).

\section{SUMMARY AND CONCLUSIONS}

Two monoalcohols (2-butanol and 2-ethyl-1-hexanol) were investigated by conventional dielectric spectroscopy and broadband shear-mechanical spectroscopy in the temperature range down to the glass-transition temperature. In the dielectric spectrum a low-frequency Debye-type process is dominant, as is generally observed for monoalcohols. The second relaxation process observed was mathematically separated from the Debye-type relaxation by assuming additivity of the processes in the dielectric susceptibility. Losspeak positions were found for the two processes.

Viewed from the shear-mechanical perspective, the liquids behave as generic glass formers. Besides a clear nonDebye alpha relaxation, a minor Johari-Goldstein beta relaxation is observed. The loss-peak positions of the alpha process were determined.

The time scale of the mechanical alpha relaxation is clearly non-Arrhenius with a fragility index of $\approx 60$ for both 

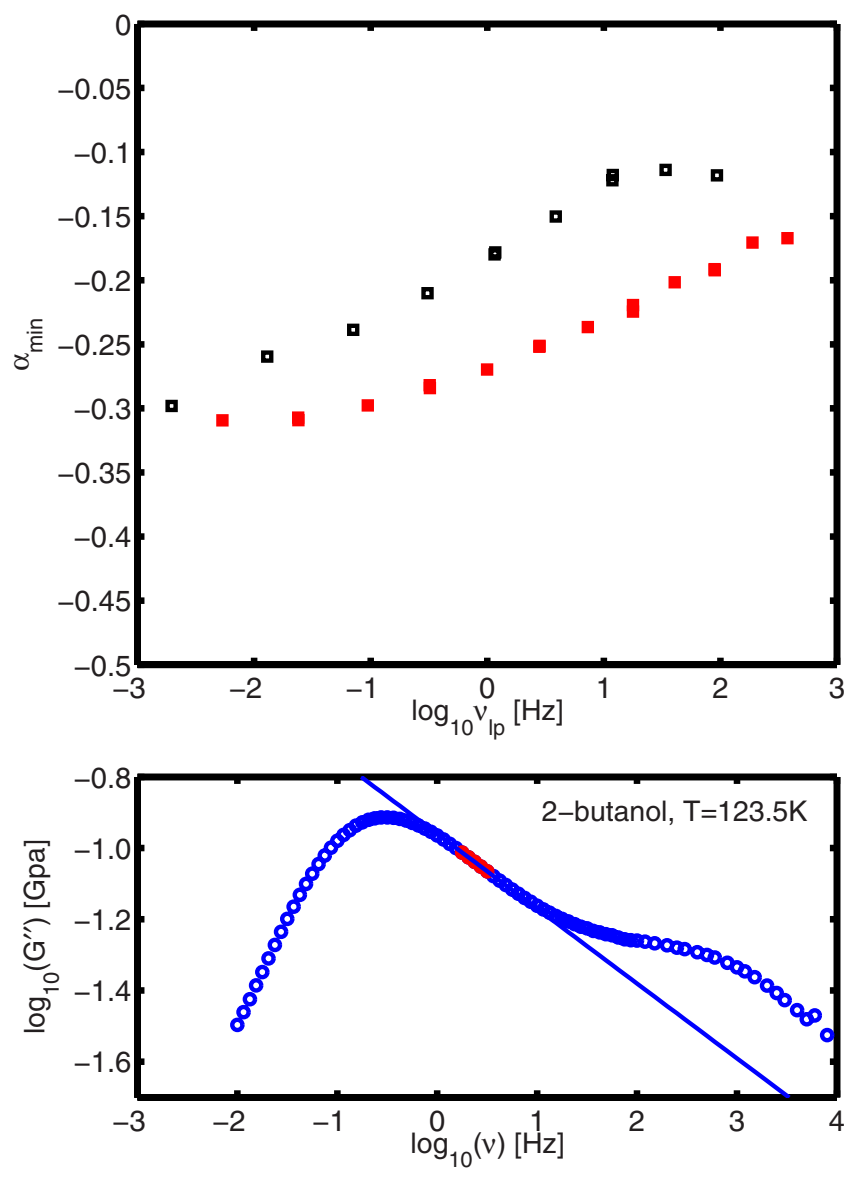

FIG. 6. (Color online) Top: minimum slope of the alpha-peak $\left(\alpha_{\min }\right)$ in a $\log -\log$ plot of $G^{\prime \prime}$ as a function of frequency, plotted against the loss-peak frequency (repeated measurements exist at some temperatures). Open symbols: 2-butanol. Closed symbols: 2-ethyl-1-hexanol. Bottom: illustration of the minimum slope in a $\log -\log$ plot of $G^{\prime \prime}$ as a function of frequency. The slope of the relaxation curve is found for all frequencies by pointwise differentiation, and from this a frequency range of minimum slope is determined. The reported minimum slope is an average over the slope in this frequency range. In the figure the frequency range of minimum slope is marked by closed circles, and the full line goes through a central point in this frequency range and has a slope equal to the found minimum slope.

liquids. The Debye-type dielectric relaxation has a much different temperature dependence, with a fragility index of $\approx 30$ for both liquids.

The time scale of the dielectric alpha relaxation follows closely that of the mechanical alpha relaxation. The two processes are separated by roughly 1 decade of frequency, consistent with what is usually observed for the separation of mechanical and dielectric alpha relaxations. The Debye-type process is separated from the mechanical alpha relaxation by roughly 4 decades (depending on temperature). The temperature dependence of the mechanical relaxation time seems to be unaffected by the falling out of equilibrium of the dielectric Debye-type process.

The possibility that the Debye-type relaxation process has a mechanical signature can still not be ruled out, but the present results show that if it exists one has to use measurement methods specialized for rather soft systems to look for it. If the Debye-type process has a mechanical signature, it must have a relaxation strength below $1 \%$ and $3 \%$ of the full relaxation strength for 2-butanol and 2-ethyl-1-hexanol, respectively.
These observations support the existing idea ${ }^{7-19}$ that the "minor" non-Debye peak observed by dielectric spectroscopy is the structural alpha relaxation, and that the major Debye-type relaxation is something else. Any explanation on the dielectric Debye-type relaxation should be able to explain why no significant signature is observed in either mechanical or calorimetric studies. ${ }^{17}$

\section{ACKNOWLEDGMENTS}

We are grateful to Niels Boye Olsen for inspiring this work and Kristine Niss for contributing with interesting comments and questions. This work was supported by the Danish National Research Foundation's (DNRF) center for viscous liquid dynamics "Glass and Time."

${ }^{1}$ C. J. F. Böttcher and P. Bordewijk, Theory of Electric Polarization II: Dielectrics in Time-Dependent Fields, 2nd ed. (Elsevier, New York, 1980).

${ }^{2}$ D. W. Davidson and R. H. Cole, J. Chem. Phys. 19, 1484 (1951).

${ }^{3}$ R. H. Cole and D. W. Davidson, J. Chem. Phys. 20, 1389 (1952).

${ }^{4}$ R. Kono, T. A. Litovitz, and G. E. McDuffie, J. Chem. Phys. 45, 1790 (1966).

${ }^{5}$ T. A. Litovitz and G. E. McDuffie, J. Chem. Phys. 39, 729 (1963).

${ }^{6}$ G. P. Johari and M. Goldstein, J. Chem. Phys. 55, 4245 (1971).

${ }^{7}$ S. S. N. Murthy, Mol. Phys. 87, 691 (1996).

${ }^{8}$ S. S. N. Murthy, J. Phys. Chem. 100, 8508 (1996).

${ }^{9}$ C. Hansen, F. Stickel, T. Berger, R. Richert, and E. W. Fischer, J. Chem. Phys. 107, 1086 (1997).

${ }^{10}$ A. Kudlik, C. Tschirwitz, S. Benkhof, T. Blochowicz, and E. Rössler, Europhys. Lett. 40, 649 (1997).

${ }^{11}$ H. Wendt and R. Richert, J. Phys. Chem. A 102, 5775 (1998).

${ }^{12}$ S. S. N. Murthy and M. Tyagi, J. Chem. Phys. 117, 3837 (2002).

${ }^{13}$ L. M. Wang and R. Richert, J. Chem. Phys. 121, 11170 (2004).

${ }^{14}$ L. M. Wang and R. Richert, J. Phys. Chem. B 109, 11091 (2005).

${ }^{15}$ L. M. Wang and R. Richert, J. Phys. Chem. B 109, 8767 (2005).

${ }^{16}$ L. M. Wang, S. Shahriari, and R. Richert, J. Phys. Chem. B 109, 23255 (2005).

${ }^{17}$ H. Huth, L. M. Wang, C. Schick, and R. Richert, J. Chem. Phys. 126, 104503 (2007).

${ }^{18}$ L. M. Wang, Y. Tian, R. Liu, and R. Richert, J. Chem. Phys. 128, 084503 (2008).

${ }^{19}$ T. El Goresy and R. Böhmer, J. Chem. Phys. 128, 154520 (2008).

${ }^{20}$ T. Lyon and T. A. Litovitz, J. Appl. Phys. 27, 179 (1956).

${ }^{21}$ B. Jakobsen, K. Niss, and N. B. Olsen, J. Chem. Phys. 123, 234511 (2005).

${ }^{22}$ W. Dannhauser and R. H. Cole, J. Chem. Phys. 23, 1762 (1955).

${ }^{23}$ S. S. N. Murthy and S. K. Nayak, J. Chem. Phys. 99, 5362 (1993).

${ }^{24}$ R. Wemelle, C. R. Hebd. Seances Acad. Sci. 244, 775 (1957).

${ }^{25}$ A. Bondeau, G. Noyel, and J. Huck, C. R. Seances Acad. Sci., Ser. C 286, 273 (1978).

${ }^{26}$ T. Christensen and N. B. Olsen, Rev. Sci. Instrum. 66, 5019 (1995).

${ }^{27}$ B. Igarashi, T. Christensen, E. H. Larsen, N. B. Olsen, I. H. Pedersen, T. Rasmussen, and J. C. Dyre, Rev. Sci. Instrum. 79, 045105 (2008).

${ }^{28}$ B. Igarashi, T. Christensen, E. H. Larsen, N. B. Olsen, I. H. Pedersen, T. Rasmussen, and J. C. Dyre, Rev. Sci. Instrum. 79, 045106 (2008).

${ }^{29}$ The full raw data sets $[G(\nu)$ and $\epsilon(\nu)$ at the investigated temperatures] can be obtained from the "Glass and Time: Data repository," found online at http://glass.ruc.dk/data.

${ }^{30}$ The problem with such approach is discussed in the context of the merging of the alpha and beta process in Ref. 47.

${ }^{31}$ K. Niss, B. Jakobsen, and N. B. Olsen, J. Chem. Phys. 123, 234510 (2005).

${ }^{32}$ N. Menon, S. R. Nagel, and D. C. Venerus, Phys. Rev. Lett. 73, 963 (1994).

${ }^{33}$ T. Christensen and N. B. Olsen, J. Non-Cryst. Solids 172-174, 357 (1994).

${ }^{34}$ R. Zorn, F. I. Mopsik, G. B. McKenna, L. Willner, and D. Richter, J. Chem. Phys. 107, 3645 (1997).

${ }^{35}$ R. D. Deegan, R. L. Leheny, N. Menon, S. R. Nagel, and D. C. Venerus, J. Phys. Chem. B 103, 4066 (1999). 
${ }^{36}$ K. Schröter and E. Donth, J. Chem. Phys. 113, 9101 (2000).

${ }^{37} \mathrm{~A}$ loss-peak position of $10^{-2} \mathrm{~Hz}$ corresponds to a relaxation time of $\approx 10 \mathrm{~s}$, a factor of 10 smaller than the often used $100 \mathrm{~s}$. The choice of relaxation time at the glass-transition temperature is always somewhat arbitrary, and will of course influence the reported glass-transition temperatures. $10^{-2} \mathrm{~Hz}$ has the advantage that equilibrium points close to and even below $T_{g}$, and are easily accessible, leading to a more precise determination of $T_{g}$.

${ }^{38}$ D. J. Plazek and K. L. Ngai, Macromolecules 24, 1222 (1991).

${ }^{39}$ R. Böhmer and C. A. Angell, Phys. Rev. B 45, 10091 (1992).

${ }^{40}$ R. Böhmer, K. L. Ngai, C. A. Angell, and D. J. Plazek, J. Chem. Phys.
99, 4201 (1993).

${ }^{41}$ O. E. Kalinovskaya and J. K. Vij, J. Chem. Phys. 112, 3262 (2000).

${ }^{42}$ C. Maggi, B. Jakobsen, T. Christensen, N. B. Olsen, and J. C. Dyre, J. Phys. Chem. B (in press).

${ }^{43}$ N. B. Olsen, T. Christensen, and J. C. Dyre, Phys. Rev. Lett. 86, 1271 (2001).

${ }^{44}$ J. C. Dyre, Europhys. Lett. 71, 646 (2005).

${ }^{45}$ J. C. Dyre, Phys. Rev. E 74, 021502 (2006).

${ }^{46}$ J. C. Dyre, Phys. Rev. E 76, 041508 (2007)

${ }^{47}$ N. Sağlanmak, K. Niss, A. I. Nielsen, J. C. Dyre, and N. B. Olsen (unpublished). 